'Departamento de Educación Física. Facultad de Educación. Universidad de Concepción. Concepción, Chile.

${ }^{2}$ Grupo IRyS. Escuela de Educación Física. Pontificia Universidad Católica de Valparaíso, Valparaíso, Chile.

${ }^{3}$ BHF Glasgow Cardiovascular Research Centre, Institute of Cardiovascular and Medical Science, University of Glasgow, Glasgow, United Kingdom. ${ }^{4}$ Departamento de Nutrición y Dietética, Facultad de Farmacia, Universidad de Concepción, Concepción, Chile. 5Instituto de Farmacia, Facultad de Ciencias, Universidad Austral de Chile, Valdivia, Chile.

${ }^{6}$ Instituto de Anatomía, Histología y Patología, Facultad de Medicina, Universidad Austral de Chile, Valdivia, Chile.

'Núcleo de investigación en Salud, Actividad

Física y Deporte, Universidad de Los Lagos, Osorno, Chile.

${ }^{8}$ Departamento de Educación Física, Deporte y Recreación, Universidad de La Frontera, Temuco, Chile.

${ }^{9}$ Departamento de Ciencias de la Actividad Física, Universidad de Los Lagos, Osorno, Chile.

${ }^{10} \mathrm{Grupo}$ de Investigación Calidad de Vida, Universidad del Biobío, Chillán, Chile.

${ }^{11}$ Departamento de Trabajo Social, INACAP, Valdivia, Chile.

${ }^{12}$ Human Nutrition Research Centre, Institute of Cellular Medicine, University of Newcastle, Newcastle upon Tyne, United Kingdom. aMagíster en Educación Física.

${ }^{\mathrm{b}}$ Doctor en Ciencias de la Actividad Física y Deportes.

'MSc. Exercise Science and Sport Medicine. dMagíster en Nutrición y Dietética 'Magíster en Neurociencias y Salud Mental. ${ }^{f}$ Magíster en Entrenamiento Deportivo. ${ }^{9}$ Doctor en Actividad Física y Salud Pública. ${ }^{h}$ Doctor en Ciencias de la Salud. 'Magíster en Educación. ITrabajadora Social.

kDoctor en Ciencias Cardiovasculares y Biomédicas.

* CS y CC-M contribuyeron de igual forma a este manuscrito y deben ser considerados como primer autor compartido.

Recibido el 28 de abril de 2016, aceptado el

11 de octubre de 2016.

Correspondencia a:

Dr. Carlos Celis-Morales

BHF Glasgow Cardiovascular Research Centre

126 University Avenue Glasgow University Glasgow

United Kingdom G12 8TA

Teléfono: +(0)44 01413304201 carlos.celis@glasgow.ac.uk

\section{Ser físicamente activo modifica los efectos nocivos del sedentarismo sobre marcadores de obesidad y cardiometabólicos en adultos}

\author{
CARLOS SALAS ${ }^{1, a^{*}}$, CARLOS CRISTI-MONTERO ${ }^{2, b^{*} \text {, }}$ \\ YU FAN ${ }^{3, \mathrm{c}}$, ELIANA DURÁN ${ }^{4, \mathrm{~d}}$, ANA MARÍA LABRAÑA ${ }^{4, \mathrm{~d}}$, \\ MARÍA ADELA MARTÍNEZ ${ }^{5, \mathrm{~d}}$, ANA MARÍA LEIVA, ${ }^{6, \mathrm{e}}$, \\ CRISTIAN ALVAREZ ${ }^{7, \mathrm{f}}$, NICOLÁS AGUILAR-FARÍAS ${ }^{8, \mathrm{~g}}$, \\ RODRIGO RAMÍREZ-CAMPILLO ${ }^{9, \mathrm{~h}}$, XIMENA DÍAZ MARTÍNEZ ${ }^{10, \mathrm{i}}$, \\ RUTH SANZANA-INZUNZA ${ }^{11, j}$, CARLOS CELIS-MORALES ${ }^{3,12, k}$
}

\section{Being physically active modifies the detrimental effect of sedentary behavior on obesity and cardiometabolic markers in adults}

Background: Sedentary behavior is a major risk factor for cardiovascular disease and mortality. Aim: To investigate whether the associations between sedentary behavior and cardiometabolic markers differs across physical activity levels. Materials and Methods: Cross sectional study of 314 participants aged 18 to 65 years. Body mass index (BMI) and waist circumference were measured, and body fat was derived from the sum of four skinfolds. Physical activity was measured objectively using accelerometers (Actigraph GT1M, USA ${ }^{\circledR}$ ). A fasting blood sample was obtained to measure glucose, insulin, HOMA-IR, lipid profile and high sensitive $C$ reactive protein (hsCRP). Those participants with an activity level $>600 \mathrm{MET}$. $\mathrm{min}^{-1}$. week ${ }^{-1}$ were classified as physically active. Results: Thirty four percent of participants were physically inactive and spent an average of 8.7 h.day $^{-1}$ in sedentary pursuits. Physically inactive individuals had poorer cardiometabolic health than their physically active counterparts. Per one hour decrease in overall sedentary behavior, there was a significant improvement in glucose (-8.46 and $-4.68{\mathrm{mg} . d l^{-1}}^{-1}$, insulin (-2.12 and -1.77 pmol. $\left.\mathrm{l}^{-1}\right)$, HOMA-IR (-0.81 and -0.56) BMI (-0.93 and $\left.-0.62 \mathrm{~kg} \cdot \mathrm{m}^{-2}\right)$ and waist circumference $(-2.32$ and $-1.65 \mathrm{~cm})$ in physically active and inactive participants, respectively. Conclusions: Being physically active may modify the detrimental effects of sedentary behavior on cardiometabolic and obesity-related traits.

(Rev Med Chile 2016; 144: 1400-1409)

Key words: Biomarkers; Physical Activity; Sedentary Lifestyle; Obesity. 
$\mathrm{M}$ undialmente, la inactividad física se ha convertido en uno de los factores de riesgo más importantes en el desarrollo de enfermedades crónicas no transmisibles ${ }^{1,2}$. La falta de actividad física (AF) causa un $9 \%$ de muertes prematuras en el mundo, equivalente a 5,3 millones de muertes por año ${ }^{1}$. Durante la última década el sedentarismo (principalmente tiempo destinado a estar sentado) ha emergido como otro importante e independiente factor de riesgo vinculado al desarrollo de obesidad, diabetes mellitus tipo 2, hipertensión arterial, síndrome metabólico, enfermedades cardiovasculares (ECVs), cáncer y mortalidad $^{2-4}$.

Si bien el sedentarismo y la inactividad física son conceptos que se utilizan frecuentemente como sinónimos debido a su estrecha relación, es muy importante clarificar que son conceptos diferentes $^{5,6}$. El sedentarismo se define como actividades asociadas a un gasto energético inferior a 1,5 METs (MET = equivalente metabólico basal; $\left.1 \mathrm{MET}=\sim 3,5 \mathrm{ml} \cdot \mathrm{kg}^{-1} \cdot \mathrm{min}^{-1}\right)$; actividades como estar sentado, ver televisión, conducir, etc. ${ }^{6}$. Mientras que ser inactivo físicamente, se asocia a no cumplir con las recomendaciones internacionales de AF, ( $\geq 600$ METs.minuto $^{-1}$. semana $^{-1}$ en adultos) $)^{6}$.

A nivel global, se estima que entre un 55\% y $70 \%$ de las actividades que se realizan diariamente (excluyendo el dormir) son de tipo sedentarias ${ }^{3,7-9}$. Los chilenos han experimentado un cambio importante en sus estilos de vida durante las últimas 3 décadas, lo que ha generado que actualmente un $19,8 \%$ de la población sea físicamente inactiva ${ }^{10}$. Sumado a esto, un $35,9 \%$ de la población destina $>4$ h al día a estar sentado ${ }^{10,11}$.

La evidencia científica ha mostrado que pasar mucho tiempo en actividades de tipo sedentarias afecta la salud independientemente del tiempo destinado a realizar $\mathrm{AF}^{3,4}$. Si bien la calidad y metodología de los estudios que describen el efecto independiente entre el sedentarismo y la AF no está en discusión, se debe señalar que la mayoría de estos trabajos han calculado el efecto nocivo del sedentarismo ajustando su análisis al tiempo destinado a realizar AF. Sin embargo, se desconoce cómo influye el nivel de sedentarismo en personas activas e inactivas físicamente sobre diversos parámetros de salud. Por tanto, el objetivo de este estudio fue investigar la asociación entre sedentarismo y marcadores cardiometabólicos, y si esta relación difiere entre sujetos que cumplen o no las recomendaciones actuales de AF.

\section{Material y Método}

\section{Diseño del estudio}

Este es un estudio observacional de corte transversal que utiliza información del proyecto GENADIO (Genes, Ambiente, Diabetes y Obesidad) realizado entre los años 2009-2011 en Chile. El estudio GENADIO tuvo como objetivo evaluar la prevalencia de factores de riesgo de ECVs en Chile, y contó con la aprobación de los Comités de Ética de la Universidad de Chile, Universidad de Concepción y Universidad de Glasgow (Reino Unido). Antes de recolectar la información todos los participantes firmaron su consentimiento informado previo a la recolección de datos.

Los participantes fueron reclutados de zonas rurales y urbanas en las regiones del Biobío y Los Ríos, siendo un $40 \%$ de estos de origen étnico mapuche, como se ha mencionado anteriormen$\mathrm{te}^{12,13}$. El cálculo del tamaño muestral de ambos estudios ha sido descrito en extenso en publicaciones previas ${ }^{12,13}$. Un total de 314 participantes fueron incluidos en el presente estudio.

La evaluación antropométrica se realizó por personal capacitado utilizando protocolos estandarizados ${ }^{12}$. El peso y la talla fueron determinados con una balanza electrónica (TANITA TBF 300A, USA) y tallímetro (SECA A800, USA) con precisión de 100 g y $1 \mathrm{~mm}$, respectivamente. El índice de masa corporal (IMC) fue calculado usando la ecuación de [peso/talla ${ }^{2}$ y el estado nutricional se definió mediante los valores de corte de la Organización Mundial de la Salud ${ }^{14}$. El perímetro de cintura fue medido con una cinta métrica no distendible (SECA, USA). Los valores utilizados para definir obesidad central fueron los siguientes: $\geq 102 \mathrm{~cm} \mathrm{y} \geq 88 \mathrm{~cm}$ en hombres y mujeres, respectivamente ${ }^{14}$. La composición corporal se determinó mediante la medición de 4 pliegues cutáneos (tricipital, bicipital, subescapular y suprailiaco) y el porcentaje de masa grasa se estimó mediante la ecuación de Durnin ${ }^{15}$.

Para la determinación de variables metabólicas se tomaron muestras de sangre venosa tras un ayuno de 8 a 12 h. Para la determinación de glicemia, colesterol total, triglicéridos (TG), colesterol HDL (HDL-c) y proteína C-reactiva ultra sensible 
(hsCRP), fueron determinados mediante métodos enzimáticos calorimétricos ${ }^{12}$. El colesterol LDL (LDL-c) fue determinado con la ecuación de Friedewald $^{16}$, la Insulina fue determinada mediante la técnica de radio-inmunoensayo, mientras que la resistencia a la insulina fue determinada mediante la ecuación de HOMA-IR ${ }^{17}$. Los coeficientes de variación fueron menor a $5 \%$ para todos los marcadores metabólicos. Todas las mediciones fueron realizadas en la Universidad de Chile y la Universidad de Glasgow ${ }^{12,13}$.

La presión arterial fue establecida como el promedio de dos mediciones realizadas después de estar 10 min en reposo, utilizando un monitor validado (OMRON HEM705, UK). Datos socio-demográficos fueron recolectados mediante encuestas validadas ${ }^{12}$.

El test de Chester fue utilizado para la medición del fitness cardiorrespiratorio (FCR) y fue expresado en METs ${ }^{18}$. Los niveles de AF y tiempo sedente fueron medidos objetivamente con acelerómetros de movimiento (Actigraph GT1M, USA). Los participantes fueron instruidos para utilizar el acelerómetro en su cadera a nivel del trocante mayor, para lo cual se les entregó una banda elástica que les permitiría fijar el monitor en esa zona. El monitor se utilizó durante 7 días y sólo se removió durante las horas destinadas a dormir, ducharse o realización de actividades acuáticas. Paralelamente, todos los participantes completaron un registro donde indicaban cuando el monitor no había sido usado y el motivo. La intensidad de la AF (sedentaria, ligera, moderada y vigorosa) fue determinada mediante el algoritmo y categorías de Freedson ${ }^{19,20}$. El tiempo destinado a AF total se estimó mediante la suma del promedio de tiempo destinado a AF ligera, moderada y vigorosa, y fue expresada en MET. $\mathrm{hr}^{-1}$.semana ${ }^{-1}$. La prevalencia de inactividad física se determinó utilizando el punto de corte de $<$ 600 MET.min ${ }^{-1}$.semana ${ }^{-1}$ de AF de intensidad moderada o vigorosa ${ }^{21}$. Por ende, participantes clasificados como físicamente activos realizaron $\geq 600 \mathrm{MET}$. $\mathrm{min}^{-1}$. semana $^{-1}$. El tiempo sedentario se determinó utilizando el punto de corte de actividades $<1,5$ METs. El tiempo sedentario se presenta como la media de horas ${ }^{-1}$. día ${ }^{-1}$ y se calcularon los treciles de sedentarismo para estratificar a la población como bajo ( $<8 \mathrm{~h}$ al día), medio (8-9,5 h al día) o alto nivel de sedentarismo (>9,5 h al día).

\section{Análisis de datos}

Se utilizó estadística descriptiva para caracterizar variables de interés según cumplimiento de las recomendaciones de AF (activo vs inactivo). La distribución normal de las variables fue analizada mediante el test de Anderson Darling y Kruskal-Wallis. Diferencias entre individuos físicamente activos e inactivos para variables cuantitativas fueron estimadas mediante General Linear Model (GLM), y las diferencias en porcentajes entre grupos fueron estimadas mediante $\chi^{2}$. La relación entre tiempo sedentario y AF fue determinada mediante el test de correlación de Pearson. La asociación entre $1 \mathrm{~h}$ de reducción en el tiempo destinado a actividades sedentarias según niveles de AF, como también la asociación entre treciles de sedentarismo y variables de tipo cardiometabólicas fueron determinadas mediante regresión múltiple, ajustando los modelos estadísticos por variables confundidoras (edad, sexo, nivel educacional y tabaquismo). Las variables metabólicas fueron ajustadas por IMC. El análisis de tendencia (P-trend) para treciles de sedentarismo en personas físicamente activas e inactivas fueron determinadas mediante la interacción entre treciles de sedentarismo y nivel de AF utilizando regresión lineal múltiple. Valor $P$ aceptado para diferencias significativas fue $<0,05$. Todos los análisis fueron realizados con el software STATA SE 14, USA.

\section{Resultados}

Las características de los participantes se presentan en la Tabla 1. En resumen, los sujetos clasificados como inactivos físicamente tenían una mayor edad, peso, IMC, porcentaje de masa grasa corporal, perímetro de cintura, tiempo de actividades sedentes y un menor FCR que el grupo físicamente activo. El grupo inactivo tenía un mayor porcentaje de personas con educación técnico-universitaria y mayor prevalencia de obesidad central. No obstante, la prevalencia de sobrepeso u obesidad (IMC $\geq 25,0)$ fue similar en ambos grupos.

Todos los marcadores metabólicos son significativamente más favorables en el grupo activo en comparación al inactivo, a excepción de la presión arterial diastólica cuyas diferencias no fueron significativas (Tabla 2). Todas estas dife- 
Tabla 1. Características socio-demográficas, antropométricas y de actividad física en grupos de personas activas e inactivas físicamente

\begin{tabular}{|c|c|c|c|}
\hline & Inactivo & Activo & $\mathbf{p}$ \\
\hline $\mathrm{n}$ & 106 & 208 & \\
\hline Edad & $39,6( \pm 13,1)$ & $36,6( \pm 12,5)$ & $<0,0001$ \\
\hline Género (hombres/mujeres) & $42 / 64$ & $95 / 113$ & 0,307 \\
\hline Tabaquismo (fumador/no fumador) & $78 / 28$ & $100 / 108$ & $<0,0001$ \\
\hline $\begin{array}{l}\text { Nivel Educacional (\%) } \\
\text { Básico } \\
\text { Enseñanza media } \\
\text { Técnico/universitario }\end{array}$ & $\begin{array}{l}20,7 \\
40,5 \\
38,6\end{array}$ & $\begin{array}{l}32,0 \\
40,2 \\
27,6\end{array}$ & 0,053 \\
\hline Peso $(\mathrm{kg})$ & $72,9( \pm 10,18)$ & $70,4 \quad( \pm 9,71)$ & 0,029 \\
\hline Talla $(\mathrm{cm})$ & $159,5( \pm 8,9)$ & $159,0( \pm 8,3)$ & 0,635 \\
\hline IMC (kg. m²) & $28,6 \quad( \pm 3,46)$ & $27,8 \quad( \pm 3,3)$ & 0,038 \\
\hline $\begin{array}{l}\text { Estado nutricional (\%) } \\
\text { Normal } \\
\text { Sobrepeso } \\
\text { Obeso }\end{array}$ & $\begin{array}{l}17,9 \\
38,6 \\
43,4\end{array}$ & $\begin{array}{l}19,2 \\
24,0 \\
56,7\end{array}$ & 0,022 \\
\hline Masa grasa \% & $30,9 \quad( \pm 3,6)$ & $28,7 \quad( \pm 4,9)$ & 0,0001 \\
\hline Perímetro de cintura $(\mathrm{cm})$ & $99,0( \pm 11,0)$ & $96,6( \pm 10,1)$ & 0,050 \\
\hline Obesidad central (\%) & 70,7 & 56,7 & $<0,0001$ \\
\hline Fitness cardiorrespiratorio (METs) & $11,7 \quad( \pm 2,7)$ & $13,9 \quad( \pm 3,5)$ & $<0,0001$ \\
\hline Actividad física (MET.hr' $\left.{ }^{-1} \cdot \mathrm{sem}^{-1}\right)$ & $12,2 \quad( \pm 4,0)$ & $16,4 \quad( \pm 4,5)$ & $<0,0001$ \\
\hline Tiempo sedentario $\left(\right.$ h. dia $\left.^{-1}\right)$ & $9,6 \quad( \pm 1,4)$ & $8,2 \quad( \pm 1,3)$ & $<0,0001$ \\
\hline
\end{tabular}

Datos presentados como media (desviación standard) para variables continuas y cómo porcentajes para variables categóricas. Individuos físicamente activos realizan $\geq 600 \mathrm{MET}$. $\mathrm{min}^{-1}$.semana ${ }^{-1}$ y sujetos físicamente inactivos $<600 \mathrm{MET}^{\mathrm{min}} \mathrm{m}^{-1}$. $\mathrm{semana}^{-1}$. En negrita se destacan los valores significativos $(p<0,05)$.

Tabla 2. Características de variables cardio-metabólicas en grupos de personas activas e inactivas físicamente

\begin{tabular}{|c|c|c|c|}
\hline & Inactivo & Activo & $\mathbf{p}$ \\
\hline PAS $(\mathrm{mmHg})$ & $126,6 \quad( \pm 18,0)$ & $121,5 \quad( \pm 15,9)$ & 0,012 \\
\hline PAD $(\mathrm{mmHg})$ & $77,4 \quad( \pm 12,4)$ & $75,7 \quad( \pm 12,3)$ & 0,258 \\
\hline Glicemia (mg. $\left.\mathrm{dl}^{-1}\right)$ & $104,7 \quad( \pm 21,9)$ & $90,3 \quad( \pm 19,8)$ & 0,0003 \\
\hline Insulina (pmol. $\left.\left.\right|^{-1}\right)$ & $10,5 \quad( \pm 9,1)$ & $5,19 \quad( \pm 5,0)$ & $<0,0001$ \\
\hline HOMA-IR & $2,71 \quad( \pm 2,3)$ & $1,26 \quad( \pm 1,3)$ & $<0,0001$ \\
\hline Colesterol total $\left(\mathrm{mg} \cdot \mathrm{dl}^{-1}\right)$ & $197,9 \quad( \pm 49,5)$ & $176,4 \quad( \pm 44,0)$ & 0,0003 \\
\hline $\mathrm{HDL}-\mathrm{c}\left(\mathrm{mg} \cdot \mathrm{dl}^{-1}\right)$ & $30,7 \quad( \pm 12,9)$ & $37,7 \quad( \pm 15,3)$ & 0,0002 \\
\hline LDL-c $\left(m g \cdot d l^{-1}\right)$ & $141,6 \quad( \pm 52,2)$ & $118,8 \quad( \pm 47,7)$ & 0,0004 \\
\hline TG $\left(m g \cdot d^{-1}\right)$ & $129,3 \quad( \pm 64,5)$ & $99,9 \quad( \pm 45,1)$ & $<0,0001$ \\
\hline $\mathrm{hsCRP}$ (mg.l-1) & $1,64 \quad( \pm 1,3)$ & $1,21 \quad( \pm 1,2)$ & 0,008 \\
\hline
\end{tabular}

Datos presentados como promedio (desviación standard). Individuos físicamente activos realizan $\geq 600 \mathrm{MET}^{\mathrm{min}} \mathrm{m}^{-1}$. semana $^{-1} \mathrm{y}$

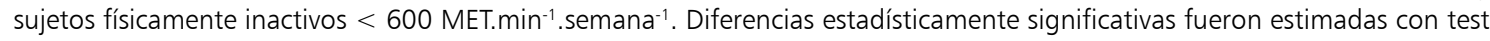
de GLM y todos los análisis fueron ajustados por edad, sexo, educación, tabaquismo e IMC. Abreviaciones: PAS: presión arterial sistólica; PAD: presión arterial diastólica; HDL-c: colesterol HDL; TG: triglicéridos, hsCRP: Proteína C-reactiva ultra sensible En negrita se aprecian los valores significativos $(p<0,05)$. 
rencias fueron independientes de la edad, sexo, educación, tabaquismo e IMC. Los niveles de sedentarismo varían entre 5,5 a $12,1 \mathrm{~h}^{-1}$.día $\mathrm{a}^{-1}$. En promedio se destinan $8,7 \mathrm{~h}^{-1}$. día $\mathrm{a}^{-1}$ a actividades de tipo sedentarias $(64,8 \%$ del día sin incluir las horas de sueño). Sólo un $4,2 \%$ y $0,3 \%$ son utilizados en AF de intensidad modera y vigorosa, respectivamente. Resultados estratificados por género se presentan en la Figura 1. Al explorar la relación entre el tiempo destinado a actividades sedentarias y el tiempo destinado a AF total (incluyendo AF de intensidad ligera, moderada y vigorosa), la correlación es significativamente negativa (Figura 2). En relación a las recomendaciones internacionales de $\mathrm{AF}\left(\geq 600\right.$ MET.min ${ }^{-1}$. semana $^{-1}$ ), un $33,8 \%$ de la población estudiada no cumple con esta dosificación, siendo las mujeres mucho más inactivas que los hombres $(36,1 \%$ versus $30,6 \%$, respectivamente).

La asociación entre el nivel de sedentarismo y marcadores metabólicos, según niveles de AF (activo vs inactivo), se presentan en la Tabla 3, Figura 3 y 4 . En resumen, por cada hora menos de tiempo destinado a actividades de tipo sedentarias, los beneficios son de mayor magnitud en el grupo activo que inactivo, tanto para variables metabólicas como de adiposidad (Tabla 3). En las Figuras 1 y 2 se presentan los resultados por treciles de sedentarismo y cumplimiento, o no, de las recomendaciones de AF. Estos resultados refle-

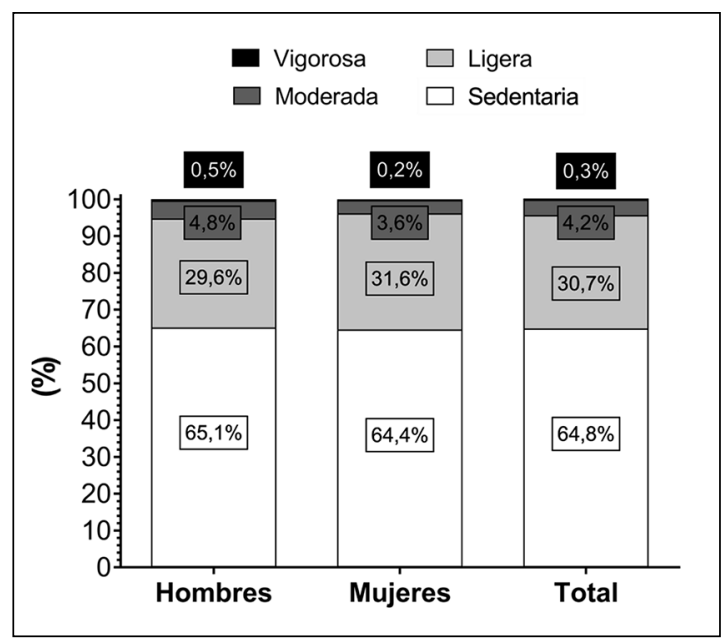

Figura 1. Distribución del tiempo destinado a diferentes intensidades de actividad física y tiempo sedente. Datos presentados como porcentaje del día sin considerar horas destinadas a dormir. jan que bajos niveles de sedentarismo se asocian a una menor adiposidad y mejor salud metabólica. No obstante, ser físicamente activo modifica el efecto nocivo del sedentarismo en marcadores de salud metabólica, tanto en niveles altos como

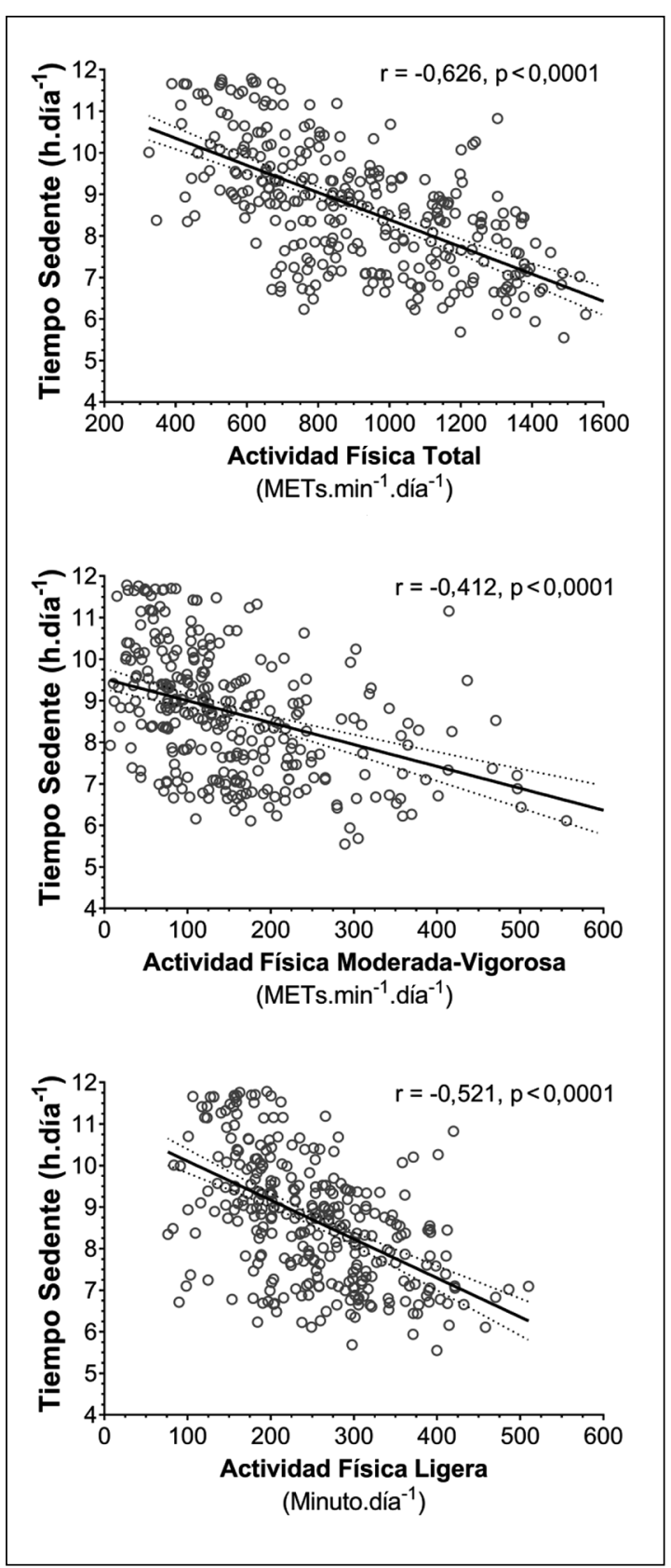

Figura 2. Correlación entre el tiempo sedentario y diferentes intensidades de actividad física. Datos son presentados como coeficiente de correlación de Pearson ( $r$ ). 
Tabla 3. Marcadores cardio-metabólicos por cada 1 hora de reducción en tiempo de sedentarismo en sujetos activos e inactivos físicamente

\begin{tabular}{|c|c|c|c|c|}
\hline & Inactivo & $\mathbf{p}$ & Activo & $\mathbf{p}$ \\
\hline Peso corporal (Kg) & $-1,13(-2,69$ a 0,43$)$ & 0,154 & $-1,93(-2,98$ a $-0,88)$ & $<0,0001$ \\
\hline IMC (m. $\left.\mathrm{kg}^{-2}\right)$ & $-0,62(-1,09 a-0,14)$ & 0,012 & $-0,93(-1,48$ a $-0,38)$ & $<0,0001$ \\
\hline Perímetro de cintura $(\mathrm{cm})$ & $-1,65(-3,20$ a $-0,09)$ & 0,038 & $-2,32(-3,39 a-1,25)$ & $<0,0001$ \\
\hline Masa grasa (\%) & $-0,35(-0,85$ a 0,15$)$ & 0,168 & $-1,04(-1,56$ a $-0,52)$ & $<0,0001$ \\
\hline PAS $(\mathrm{mmHg})$ & $-0,92(-3,59$ a 1,75$)$ & 0,496 & $-1,69(-3,40$ a 0,08$)$ & 0,051 \\
\hline PASD $(\mathrm{mmHg})$ & $-0,76(-2,70$ a 1,17$)$ & 0,437 & $-1,35(-2,70$ a $-0,04)$ & 0,017 \\
\hline Glicemia (mg. $\mathrm{dl}^{-1}$ ) & $-4,68(-8,1 a-1,26)$ & 0,006 & $-8,46(-13,5$ a $-3,42)$ & 0,001 \\
\hline Insulina (pmol. $\left.\left.\right|^{-1}\right)$ & $-1,77(-2,78$ a $-0,77)$ & 0,001 & $-2,12(-2,71$ a $-1,53)$ & $<0,0001$ \\
\hline HOMA-IR & $-0,56(-0,80$ a $-0,32)$ & $<0,0001$ & $-0,81(-1,24$ to $-0,38)$ & $<0,0001$ \\
\hline Colesterol total $\left(\mathrm{mg} \cdot \mathrm{dl}^{-1}\right)$ & $1,59(-5,22$ a 8,42$)$ & 0,642 & $-4,63(-8,93$ a $-0,33)$ & 0,037 \\
\hline $\mathrm{HDL}-\mathrm{c}\left(\mathrm{mg} \cdot \mathrm{dl}^{-1}\right)$ & $1,76(-0,10$ a 3,63$)$ & 0,064 & $2,05(0,31$ a 3,79$)$ & 0,010 \\
\hline LDL-c (mg.dl-1) & $0,56(-6,78$ a 7,92$)$ & 0,878 & $-4,74(-9,02$ a $-0,46)$ & 0,047 \\
\hline $\mathrm{TG}\left(\mathrm{mg} \cdot \mathrm{dl}^{-1}\right)$ & $-3,71(-13,5$ a 3,14$)$ & 0,455 & $-6,68(-12,1 \mathrm{a}-1,31)$ & 0,015 \\
\hline hsCRP (mg. $\left.\mathrm{l}^{-1}\right)$ & $-0,11(-0,31$ a $-0,9)$ & 0,303 & $-0,24(-0,39 a-0,09)$ & 0,035 \\
\hline
\end{tabular}

Datos presentados como coeficiente beta y su respectivo $95 \%$ de intervalo de confianza. Individuos físicamente activos realizan $\geq 600 \mathrm{MET} \cdot \mathrm{min}^{-1}$. semana-1 y sujetos físicamente inactivos < $600 \mathrm{MET}$. min ${ }^{-1}$.semana-1 . Asociaciones estadísticamente significativas fueron estimadas con regresión lineal múltiple y todos los análisis fueron ajustados por edad, sexo, educación, tabaquismo. Variables metabólicas también fueron ajustadas por IMC. Abreviaciones: PAS: presión arterial sistólica; PAD: presión arterial diastólica; HDL-c: colesterol HDL; TG: triglicéridos, hsCRP: Proteína C-reactiva ultra sensible. En negrita se aprecian los valores significativos $(p<0,05)$.

en niveles bajos de sedentarismo. Niveles más bajos de sedentarismo, es decir, estar en un nivel alto, medio o bajo, se asocia a un menor riesgo metabólico en el grupo de personas físicamente activas, sin embargo, este efecto no parece influir de la misma manera en el grupo inactivo (Figura 4). Estas asociaciones también se observaron para marcadores de adiposidad (Figura 3).

\section{Discusión}

\section{Principales hallazgos}

Los principales resultados de este estudio señalan que ser físicamente activo modifica los efectos nocivos del sedentarismo sobre marcadores de adiposidad y salud cardiometabólica; no encontramos otro trabajo publicado con estas características realizado en el país. Estas asociaciones fueron independientes de variables confundidoras como la edad, género, tabaquismo, educación e IMC.

\section{Comparación y relación con estudios previos}

Nuestros resultados concuerdan con los únicos dos estudios que se han realizado hasta la fecha ${ }^{22,23}$. Bakrania y cols. ${ }^{22}$, reportaron en un estudio de 2.131 adultos que individuos físicamente activos pero con altos niveles de sedentarismo presentaban un mejor perfil metabólico y de adiposidad que aquellos inactivos y con altos niveles de sedentarismo. Resultados similares fueron presentados por Loprinzi y cols. ${ }^{23}$, en un estudio de la encuesta NHANES 2003-2006 con 5.580 adultos, quienes reportaron que las personas físicamente activas presentan menores niveles de insulina y glucosa, independiente del tiempo destinado a actividades sedentarias.

La práctica sistemática de ejercicio físico y la reducción del tiempo sedentario son estrategias de prevención que han demostrado influir positivamente sobre diversos parámetros de salud ${ }^{3,4,24,25} \mathrm{y}$ mortalidad ${ }^{26}$; sin embargo, hasta el día de hoy en nuestro país, no se les ha otorgado la importancia 


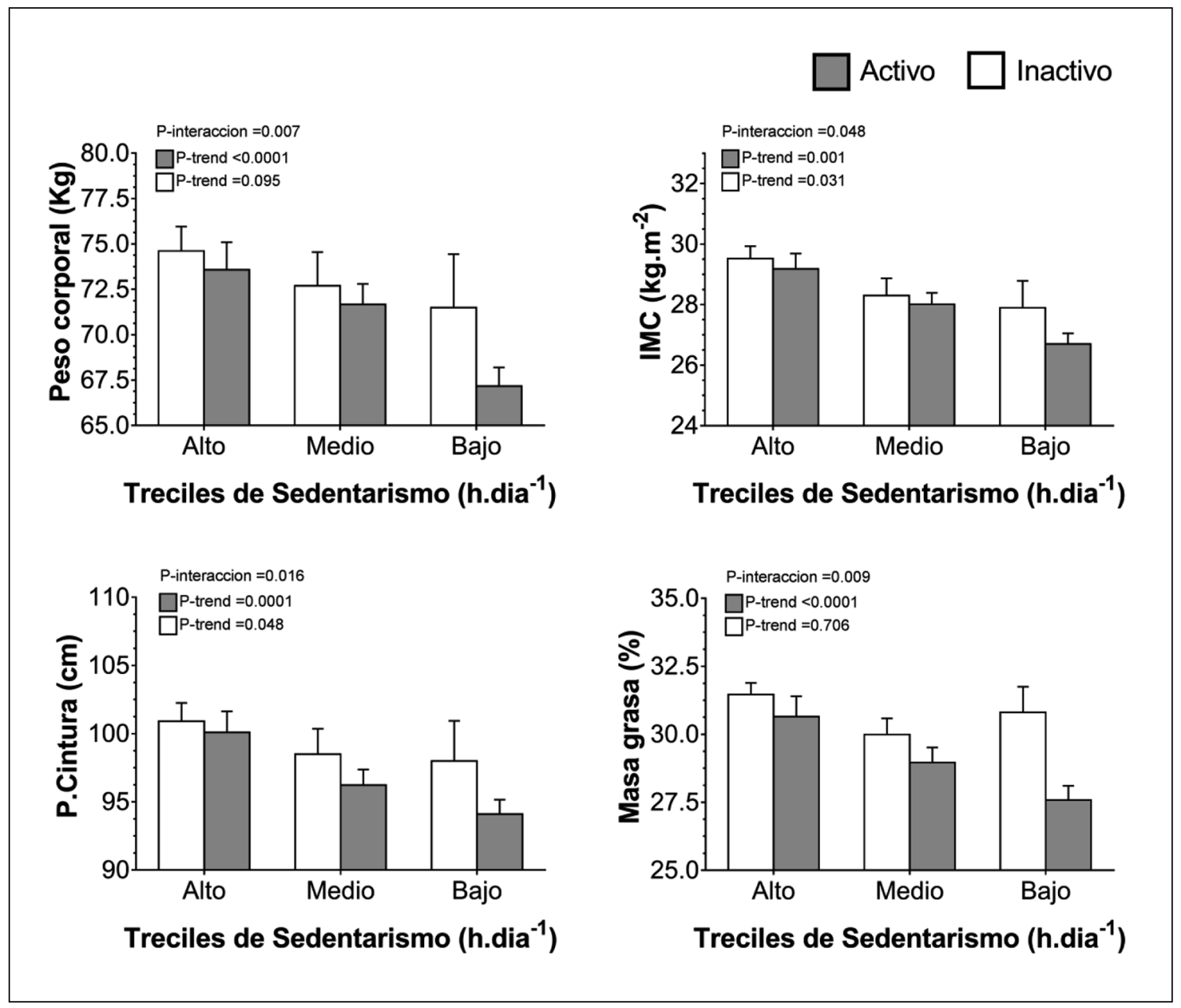

Figura 3. Asociación entre niveles de sedentarismo y marcadores de adiposidad según niveles de actividad física. Datos presentados como promedio y error estándar de la media. Barras grises representan individuos físicamente activos (realizan $\geq 600$ MET. $\mathrm{min}^{-1}$. semana ${ }^{-1}$ ) y barras blancas representan sujetos inactivos (< $600 \mathrm{MET}$. $\mathrm{min}^{-1}$. semana $^{-1}$ ). El valor-p estimado representa la tendencia (P-trend). Todos los análisis fueron ajustados por edad, sexo, educación y tabaquismo.

que merece tanto a nivel clínico como de salud pública.

Al respecto, un estudio experimental de Duvivier et al., (2013) que comparó un grupo de sujetos activos ( 1 h de ejercicio al día por 4 días) y otro de inactivos físicamente que debían romper el hábito sedentario (poniéndose de pie y/o caminado un período de tiempo equivalente energéticamente a $1 \mathrm{~h}$ de ejercicio al día), demostró que este segundo grupo logró mejores resultados en la acción de la insulina y lípidos plasmáticos que el primero ${ }^{24}$. Estos resultados, demuestran la importancia de romper el hábito sedentario y mantener un nivel de AF constante durante todo el día y no sólo durante un breve período de tiempo (ejemplo, 45 min de ejercicio equivale al $~ 5 \%$ del tiempo de vigilia total del día de una persona $)^{25}$.

\section{Fortalezas y limitaciones del estudio}

Entre las fortalezas de este estudio se encuentra la medición objetiva de la AF y sedentarismo (acelerometría), lo cual permite reducir el sesgo asociado a datos recolectados a través de cuestionarios ${ }^{13}$. Una de las limitaciones del presente estudio tiene relación a que la muestra incorporada no es necesariamente representativa de la población 
Activo $\square$ Inactivo

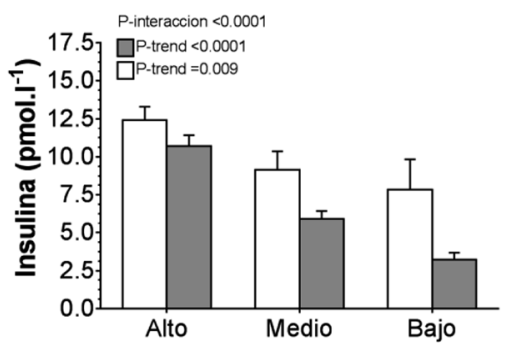

Treciles de Sedentarismo (h.dia ${ }^{-1}$ )
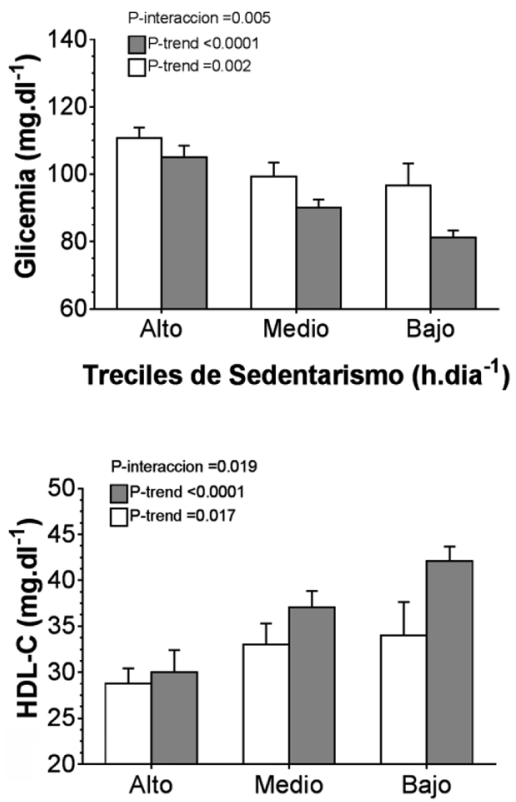

Treciles de Sedentarismo (h.dia $\left.{ }^{-1}\right)$

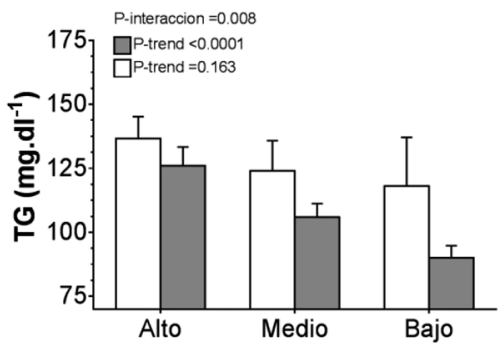

Treciles de Sedentarismo (h.dia ${ }^{-1}$ )

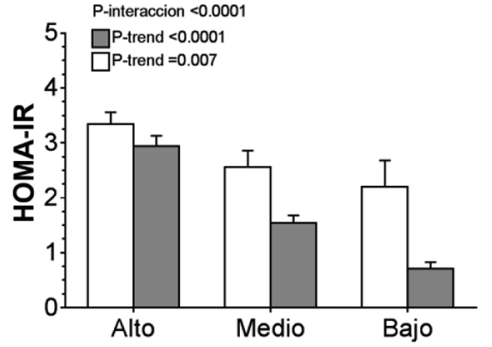

Treciles de Sedentarismo (h.dia ${ }^{-1}$ )

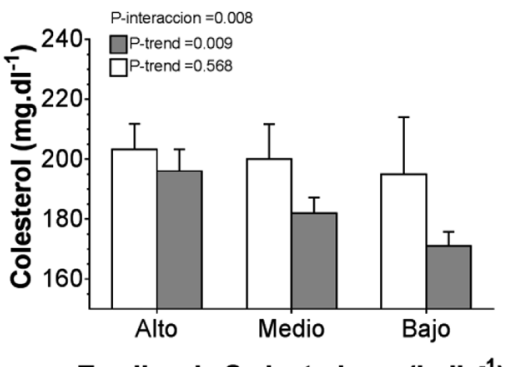

Treciles de Sedentarismo (h.dia ${ }^{-1}$ )

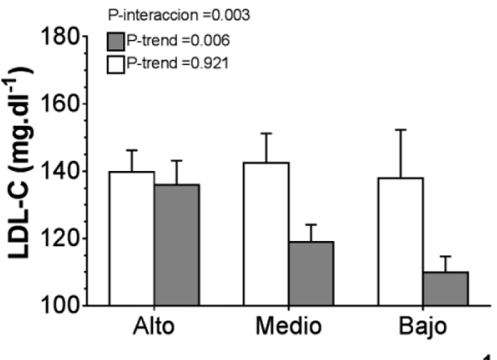

Treciles de Sedentarismo (h.dia ${ }^{-1}$ )

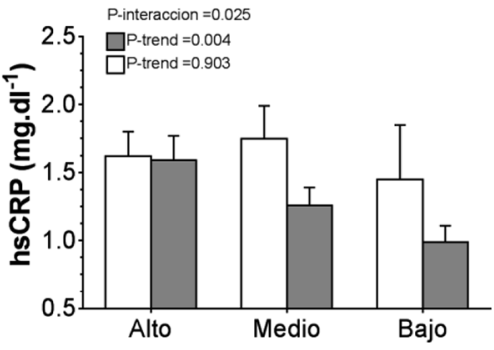

Treciles de Sedentarismo (h. dia ${ }^{-1}$ )

Figura 4. Asociación entre niveles de sedentarismo y marcadores metabólicos según niveles de actividad física. Datos presentados como promedio y error estándar de la media. Barras grises representan individuos físicamente activos (realizan $\geq 600$ MET. $\mathrm{min}^{-1}$. semana ${ }^{-1}$ ) y barras blancas representan sujetos inactivos ( $<600 \mathrm{MET}$. $\mathrm{min}^{-1}$. semana $\left.^{-1}\right)$. El valor-p estimado representa la tendencia (P-trend). Todos los análisis fueron ajustados por edad, sexo, educación, tabaquismo elMC. 
nacional, por ende futuros estudios, y principalmente encuestas de carácter nacional, deberían incluir la medición objetiva de actividad física, de manera de obtener una mejor cuantificación del perfil epidemiológico de actividad física del país. El diseño de corte transversal de este estudio no permite establecer asociaciones de causa-efecto, por lo cual, estudios de intervención son necesarios para establecer este tipo de asociaciones.

\section{Implicaciones de los resultados}

Los mayores beneficios en marcadores cardio-metabólicos se observan en personas activas con niveles medios o bajos de sedentarismo. Mientras que las personas activas ubicadas en el trecil más alto de sedentarismo ("mayor riesgo") no presentan una gran diferencia en comparación al grupo físicamente inactivo. Lo señalado demuestra la importancia que tiene cumplir con las recomendaciones de AF para controlar y revertir el riesgo de desarrollo de enfermedades y que los efectos beneficiosos del ejercicio físico podrían verse mermados por un exceso de tiempo sedentario ${ }^{27}$.

Si se consideraran los resultados obtenidos en el presente estudio y se tomara en cuenta que una gran parte de la población chilena realiza trabajos $o$ actividades en tiempo libre que involucran una gran cantidad de tiempo sedente (ej., trabajo de oficina, frente a un computador, ver televisión, etc.), se hace imprescindible que futuras intervenciones y políticas públicas (a nivel de salud, educación, transporte, etc.) centren sus esfuerzos no sólo en incrementar los niveles de AF de los chilenos, sino también en reducir los niveles de sedentarismo.

\section{Conclusiones}

Los resultados del presente estudio muestran que ser físicamente activo modifica en gran medida los efectos nocivos de ser sedentario sobre marcadores cardiometabólicos. Disminuir el tiempo destinado a actividades de bajo costo energético, como pasar mucho tiempo sentado, debiera ser un complemento indispensable a las actuales recomendaciones de AF para nuestra población.

Agradecimientos: Se agradece de forma especial al Dr. Francisco Pérez, Dra. Natalia Ulloa y Dr. Carlos Calvo por su contribución en la etapa de recolección de datos en este estudio. También se agradece al Dr. Jason Gill y Dr. Mark Bailey por su contribución en el diseño del estudio. Carlos Celis-Morales concibió la pregunta de investigación. Carlos Celis-Morales realizo los análisis estadísticos. Carlos Salas y Carlos Celis-Morales redactaron el manuscrito. CCM, ED, AML, MAD, AML, CA, NF, RRC, XDM y RSI revisaron críticamente el manuscrito. Todos los autores aprobaron la versión final del manuscrito.

\section{Referencias}

1. WHO. Global recommendations on physical activity for health: World Health Organization, 2010.

2. Celis-Morales C, Lyall DM, Anderson J, Pell JP, Sattar N, Gill J. The association between physical activity and risk of mortality is modulated by grip strength and cardiorespiratory fitness: evidence from 498,135 UK-Biobank participants. Eur Heart J 2016; Jul 6. pii: ehw249.

3. Owen N, Healy GN, Matthews CE, Dunstan DW. Too Much Sitting: The Population Health Science of Sedentary Behavior. Exerc Sport Sci Rev 2010; 38 (3): 105-13.

4. Dunstan DW, Howard B, Healy GN, Owen N. Too much sitting-A health hazard. Diabetes Res Clin Pract 2012; 97 (3): 368-76.

5. Cristi-Montero C, Rodríguez FR. The paradox of being physically active but sedentary or sedentary but physically active. Rev Med Chile 2014; 142 (1): 72-8.

6. Cristi-Montero C, Celis-Morales C, Ramírez-Campillo R, Aguilar-Farías N, Álvarez C, Rodríguez-Rodríguez F. Sedentary behaviour and physical inactivity is not the same!: An update of concepts oriented towards the prescription of physical exercise for health. Rev Med Chile 2015; 143 (8): 1089-90.

7. Matthews CE, Chen KY, Freedson PS, Buchowski MS, Beech BM, Pate RR, et al. Amount of time spent in sedentary behaviors in the united states, 2003-2004. Am J Epidemiol 2008; 167 (7): 875-81.

8. Colley RC, Garriguet D, Janssen I, Craig CL, Clarke J, Tremblay MS. Physical activity of Canadian adults: accelerometer results from the 2007 to 2009 Canadian Health Measures Survey. Health Rep 2011; 22 (1): 7-14.

9. Bennie JA, Chau JY, van der Ploeg HP, Stamatakis E, Do A, Bauman A. The prevalence and correlates of sitting in European adults - a comparison of 32 Eurobarometer-participating countries. Int J Behav Nutr Phys Act 2013; $10: 107$.

10. Celis-Morales C, Salas C, Alduhishy A, Sanzana R, Martínez MA, Leiva A, et al. Socio-demographic patterns of physical activity and sedentary behaviour in Chile: 
results from the National Health Survey 2009-2010. J Public Health 2016; 38 (2): e98-e105.

11. Celis-Morales C, Salas C, Álvarez C, Aguilar Farías N, Ramírez Campillos R, Leppe J, et al. Higher physical activity levels are associated with lower prevalence of cardiovascular risk factors in Chile. Rev Med Chile 2015; 143 (11): 1435-43.

12. Celis-Morales CA, Pérez-Bravo F, Ibanes L, Sanzana R, Hormazábal E, Ulloa N, Calvo C, et al. Insulin Resistance in Chileans of European and Indigenous Descent: Evidence for an Ethnicity x Environment Interaction. Plos One 2011; 6 (9).

13. Celis-Morales CA, Pérez-Bravo F, Ibáñez L, Salas C, Bailey ME, Gill JM. Objective vs self-reported physical activity and sedentary time: effects of measurement method on relationships with risk biomarkers. PloS one 2012; 7 (5): e36345.

14. WHO. Obesity: preventing and managing the global epidemic. Report of a WHO consultation. World Health Organization technical report series 2000; 894: i-xii, 1-253.

15. Durnin J, Womersle. J. Body fat assessed fro, total-body density and its estimation from skidfold thickness: measurement on 481 men and women aged from 16 to 72 years. Br J Nutr 1974; 32 (1): 77-97.

16. Friedewald WT, Levy RI, Fredrickson DS. Estimation of concentration of low-density lipoprotein cholesterol in plasma, without use of preparative ultracentrifuge. Clin Chem 1972; 18 (6): 499-502.

17. Matthews DR, Hosker JP, Rudenski AS, Naylor BA, Treacher DF, Turner RC. Homeostasis model assessment: insulin resistance and beta-cell function from fasting plasma glucose and insulin concentrations in man. Diabetologia 1985; 28 (7): 412-9.

18. Buckley JP, Sim J, Eston RG, Hession R, Fox R. Reliability and validity of measures taken during the Chester step test to predict aerobic power and to prescribe aerobic exercise. Br J Sports Med 2004; 38 (2): 197-205.

19. Freedson P, Bowles HR, Troiano R, Haskell W. Assessment of physical activity using wearable monitors: recommendations for monitor calibration and use in the field. Med Sci Sports Exerc 2012; 44 (1 Suppl 1): S1-4.

20. Freedson PS, Melanson E, Sirard J. Calibration of the Computer Science and Applications, Inc. accelerometer. Med Sci Sports Exerc 1998; 30 (5): 777-81.

21. WHO. Global Physical Activity Questionnaire: GPAQ version 2.0: World Health Organization, 2009.

22. Bakrania K, Edwardson CL, Bodicoat DH, Esliger DW, Gill JM, Kazi A, et al. Associations of mutually exclusive categories of physical activity and sedentary time with markers of cardiometabolic health in English adults: a cross-sectional analysis of the Health Survey for England. BMC Public Health 2016; 16 (1): 25.

23. Loprinzi PD, Lee H, Cardinal BJ. Daily movement patterns and biological markers among adults in the United States. Prev Med 2014; 60: 128-30.

24. Duvivier BMFM, Schaper NC, Bremers MA, van Crombrugge G, Menheere PP, Kars M, et al. Minimal Intensity Physical Activity (Standing and Walking) of Longer Duration Improves Insulin Action and Plasma Lipids More than Shorter Periods of Moderate to Vigorous Exercise (Cycling) in Sedentary Subjects When Energy Expenditure Is Comparable. Plos One 2013; 8 (2).

25. Smith L, Ekelund U, Hamer M. The Potential Yield of Non-Exercise Physical Activity Energy Expenditure in Public Health. Sports Med 2015; 45 (4): 449-52.

26. Katzmarzyk PT. Standing and Mortality in a Prospective Cohort of Canadian Adults. Med Sci Sports Exerc 2014; 46 (5): 940-6.

27. Kulinski JP, Khera A, Ayers CR, Das SR, de Lemos JA, Blair SN, et al. Association Between Cardiorespiratory Fitness and Accelerometer-Derived Physical Activity and Sedentary Time in the General Population. Mayo Clin Proc 2014; 89 (8): 1063-71. 